\title{
A POLÍTICA DE AMPLIAÇÃO DA COBERTURA DO SNS NA SAÚDE ORAL E VISUAL EM PORTUGAL
}

\author{
Denise Capela dos Santos
}

ORCID ID:0000-0002-5794-5203;

CIÊNCIA ID: 0B10-1C58-71EB,

dsantos@autonoma.pt

O Orçamento de Estado (OE) 2017 define como prioritário o investimento em "novas respostas no âmbito dos Meios Complementares de Diagnóstico e Terapêutica (MCDT) nas unidades de cuidados de saúde primários e alargamento das experiências no âmbito da saúde oral e visual" (República Portuguesa, 2016) ${ }^{a}$ da população. No entanto, no âmbito do Plano Nacional de Saúde, a saúde oral e a saúde visual não foram consideradas na atribuição de Programas de

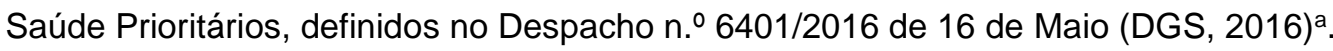

Todavia, devido à elevada prevalência de patologias nestas áreas em todo o Mundo, associadas a significativa incapacidade quando o tratamento é negligenciado e atendendo à débil resposta que o Serviço Nacional de Saúde (SNS) tem dado face às necessidades da população em saúde oral e visual, é importante que haja uma ampliação da cobertura do SNS, nestas áreas, no menor espaço de tempo, em qualquer parte do território.

Sem recursos para responder às necessidades nesta matéria, o SNS tem pressionando a população para o acesso ao setor privado, onde as consultas e os tratamentos são particularmente caros e inalcançáveis por uma grande parte da população.

Não nos podemos esquecer que, em Portugal, $26,6 \%$ das pessoas vive em situação de pobreza e de exclusão social (EAPN Portugal, 2016).

O planeamento da ampliação da oferta de cuidados em saúde oral e visual nos Cuidados de Saúde Primários (CSP) é assim muito bem-vindo e acredita-se que procurará assegurar a adequada cobertura territorial, reforçar do número de vagas na rede em medicina dentária e estomatologia e em oftalmologia, privilegiando o aproveitamento dos recursos disponíveis, num esforço conjunto entre Agrupamentos de Centros de Saúde (ACES)/ Unidades Locais de Saúde (ULS), hospitais e o setor privado, com especial incidência nos grandes centros urbanos. 
Working Paper

Departamento de Ciências Económicas e Empresariais

Universidade Autónoma de Lisboa

\section{SAÚDE ORAL}

No Despacho n.- 8591-B/2016, publicado em Diário da República a 1 de Julho, executou-se uma Revisão do Programa Nacional de Promoção de Saúde Oral, no âmbito dos cuidados de saúde primários (SNS, 2016) $)^{b}$, tendo o Estado optado por investir na ampliação da cobertura do Serviço Nacional de Saúde (SNS) em saúde oral. Esta revisão inclui também o acesso a CSP, através da promoção da saúde, da prevenção da doença, da disponibilização de consultas de saúde oral nos ACES, e a atribuição de cheques-dentista, nos termos da Portaria n.․ 301/2009, de 24 de Março, quando o SNS não tem capacidade de resposta (SNS, 2016) .

Para conseguir alcançar os objetivos a que se tinha proposto, o Governo lançou-se num projeto piloto, de implementação de consultas de saúde oral em 13 Centros de Saúde em Lisboa e no Alentejo, no primeiro semestre do ano de 2016, em colaboração com a Ordem dos Médicos Dentistas (Jornal Médico, 2016)a.

Numa primeira fase, serão contratados médicos dentistas para:

- ACES Almada -Seixal: Centro de Saúde do Monte Caparica;

- ACES Arco Ribeirinho: Centro de Saúde da Moita;

- ACES Médio Tejo: Centro de Saúde de Fátima;

- ACES Lezíria: Centro de Saúde de Salvaterra de Magos, Centro de Saúde do Cartaxo, Centro do Saúde de Rio Maior;

- ACES Estuário Tejo: Centro de Saúde da Azambuja, Centro de Saúde de Alenquer, Centro de Saúde da Arruda dos Vinhos;

- ACES Oeste Sul: Centro de Saúde da Lourinhã, Centro de Saúde Mafra Ericeira;

- ACES Alentejo Central: Centro de Saúde de Montemor-o-Novo, Centro de Saúde de Portel (DGS, 2016) .

Estes Centros de Saúde foram escolhidos por terem equipamentos e higienistas orais, embora os edifícios tenham de ser adaptados para este fim (Público, 2016) .

De facto, as experiências-piloto com dentistas nos Centros de Saúde arrancaram em Março em 13 Unidades do Alentejo e de Lisboa e o Governo prevê que, até 2020, passem a 91 (Público, 2016) a USF.

Na primeira fase do projeto, que decorre até 31 de Dezembro de 2016, têm apenas acesso a consultas de saúde oral "os doentes portadores de diabetes, neoplasias, patologia cardíaca ou respiratória crónica, insuficiência renal em hemodiálise ou diálise peritoneal e os transplantados, inscritos nos ACES, onde decorrem as experiências piloto", privilegiando -se os utentes com menor capacidade económica (SNS, 2016) ${ }^{\text {b }}$. Só na segunda fase, a partir de 1 de janeiro de 2017, o projeto poderá ser alargado, de forma faseada, a todos os utentes inscritos nos ACES onde decorrem as experiências-piloto, dependendo da referenciação pelo médico de família e dos recursos disponíveis (SNS, 2016) . Sugere-se portanto uma avaliação sustentada das necessidades não salvaguardadas da população, principalmente da mais carenciada, de forma 
Working Paper

Departamento de Ciências Económicas e Empresariais

Universidade Autónoma de Lisboa

a que seja mais fácil adequar um planeamento orçamental e planeamento de recursos para prestação de cuidados, às necessidades reais.

Outra medida importante para a Saúde Oral é o Programa Nacional para a Promoção da Alimentação Saudável (PNPAS). Como é sabido, hábitos alimentares inadequados incluem a ingestão excessiva de açúcares simples, cujo consumo constitui um dos principais fatores de risco para as cáries dentárias, uma das doenças não transmissíveis mais prevalentes a nível Mundial (DGS, 2016).

Segundo a OMS, o consumo de açúcar pelas crianças Portuguesas aproxima-se dos $25 \%$ da energia ingerida diariamente, ou seja cinco vezes acima dos $5 \%$ ideais (DGS, 2016) .

Este PNPAS é importante porque promove a redução do consumo de açúcar em Portugal e é apoiado pela Secretária-Geral da Associação Industrial e Comercial do Café (DGS, 2016) ${ }^{c}$. Uma das medidas tomadas pela Direcção-Geral da Saúde para este efeito foi a proposta de redução dos atuais oito gramas de açúcar das embalagens individuais servidas na restauração para apenas quatro ou três grama, sugerindo ainda, que seja obrigatório que os pacotes de açúcar sejam apenas disponibilizados aos clientes que o pedirem (DGS, 2016) ${ }^{\mathrm{d}}$. Concorda-se plenamente com a medida, acreditando que a toxicidade associada à ingestão de açúcar está diretamente relacionada com a redução de patologias ao nível da boca, e de outras de importância considerável no território nacional, como a diabetes e a obesidade.

Numa tentativa de perceber a dimensão do problema, a Direção-Geral da Saúde apresentou em 2006, o terceiro Estudo Epidemiológico Nacional das Doenças Orais, desenvolvido em parceria com o Programa Nacional de Promoção da Saúde Oral e com a Ordem dos Médicos Dentistas. Neste estudo, foi inquirida uma amostra de 6315 indivíduos, de 2012 a 2014, representativa a nível nacional (DGS, 2016) .

Alcançaram-se os seguintes resultados:

1. "Redução do índice CPOD (dentes cariados, perdidos e obturados) que, aos 12 anos, era de 1,48, em 2006, e passou para 1,18 em 2013;

2. Aumento da percentagem de jovens com gengivas saudáveis que em 2006, aos 12 anos era $29 \%$ e aos 15 anos era $22 \%$, enquanto em 2013, aos 12 anos era $51,7 \%$ e aos 18 anos $41,8 \%$

3. Aumento do número médio de dentes com selantes de fissura que, em 2006, aos 12 anos era 1,60 e aos 15 anos era 0,86 e, em 2013, aos 12 anos era 3,61 e aos 18 anos era 1,31 ;

4. Aumento da percentagem de crianças e jovens que escovam os dentes à noite, antes de deitar, que em 2006, aos 6 anos era de 35\%, aos 12 anos $51 \%$ e aos 15 anos era de $45 \%$, enquanto em 2013 aos 6 anos essa percentagem era de $84 \%$, aos 12 anos, $87 \%$ e aos 18 anos 84\%" (DGS, 2016) 
Working Paper

Departamento de Ciências Económicas e Empresariais

Universidade Autónoma de Lisboa

Os resultados indicam que a saúde oral das crianças e jovens melhorou. É, no entanto, importantíssimo avaliar a prevalência de CPOD, estado das gengivas e capacidade de escovagem de dentes nos idosos isolados e carenciados. Não se encontraram dados neste grupo socio-económico.

\section{SAÚDE VISUAL}

Em Portugal existe um Programa Nacional para a Saúde da Visão (PNSV), de 2005, onde está descrita a estratégia para execução de exames oftalmológicos à população, com elevado risco de desenvolvimento de patologia visual, com base na sua história clínica, familiar e idade, mesmo quando não existe uma sintomatologia associada. Este programa prevê a elaboração de, pelo menos, uma observação oftalmológica das crianças com idades entre os 0-2 anos e outra entre os 2-5 anos (SNS, 2016) .

O atual Governo decidiu, de forma pioneira, através do Despacho n. ${ }^{\circ}$ 5868-B/2016, publicado no Diário da República de 2 de Maio (SNS, 2016)a , implementar, no primeiro semestre de 2016 e no âmbito do PNSV, o Rastreio de Saúde Visual Infantil (RSVI), assim com o rastreio da Degenerescência Macular da Idade (DMI) em diabéticos (Jornal Médico, 2016)a , associando este rastreio ao da Retinopatia Diabética para adultos, que era já prática corrente nos CSP.

O RSVI pressupõe a observação de todas as crianças no semestre em que completam dois anos, e de outro rastreio a todas as crianças, entre os 4 e os 5 anos de idade (SNS, 2016) ${ }^{\text {a }}$ O rastreio visual precoce é fulcral, porque, se detetada a tempo, a ambliopia pode ser revertida e evitada uma eventual cegueira (Público, 2016) , revelando-se a prevenção da doença na infância essencial para a saúde visual da população ao longo da vida.

Quanto às restantes patologias, o Observatório Nacional da Diabetes estima a prevalência da Diabetes, entre os 20 e os 79 anos, em 13,1\%, a que corresponde cerca de 1 milhão de Portugueses, dos quais aproximadamente 400 mil não estão diagnosticados (Genro, 2016). A Retinopatia Diabética é a principal complicação a nível ocular desta doença e pode evoluir sem apresentar nenhuma sintomatologia, sendo uma das principais causas de perda grave de visão a nível Mundial. Após 20 anos de ocorrência de doença, mais de $90 \%$ das pessoas com diabetes tipo 1 e mais de $60 \%$ das de tipo 2 sofrem de retinopatia diabética (Jornal Médico, 2016) ${ }^{\text {b. No }}$ ano de 2014, segundo dados deste Observatório, foram rastreados para a Retinopatia Diabética 95.535 indivíduos diabéticos (cerca de 10\% do total de doentes em Portugal). Nesta amostra, foi possível identificar 8.320 indivíduos a necessitar de encaminhamento para centro especializado, com a colaboração ativa da Associação Protetora dos Diabéticos de Portugal (APDP) (Genro, 2016). Considera-se que este é um rastreio de grande importância para a população. 
A associação do Rastreio da DMI ao Rastreio da Retinopatia Diabética é também fundamental, considerando que a DMI é a causa mais frequente de cegueira nos países desenvolvidos em pessoas com mais de 55 anos. Como o principal fator de risco associado é a idade, espera-se uma prevalência crescente da doença no Mundo Ocidental, atendendo ao aumento da esperança média de vida nestes países.

A periodicidade do rastreio da DMI é anual e calcula-se que, a partir de 1 de Julho, sejam rastreados 37.412 diabéticos com mais de 55 anos de quatro ACES (Público, 2016) ${ }^{\text {. }}$.

As experiências-piloto em Saúde Visual (rastreio da DMI e ambliopia em crianças) arrancaram, em Maio de 2016, em quatro ACES do Porto (Público, 2016)a e em dois Centros Hospitalares.

- $\quad$ ACES Grande Porto V - Porto Ocidental

- $\quad$ ACES Grande Porto VI - Porto Oriental

- ACES Grande Porto II - Gondomar

- ACES Grande Porto III - Maia e Valongo

- $\quad$ Centro Hospitalar de São João, EPE

- $\quad$ Centro Hospitalar do Porto, EPE (SNS, 2016)a.

Todas as crianças da faixa etária descrita, a residir em território nacional que tenham feito o RSVI, e todos os utentes diabéticos do SNS selecionados para o rastreio primário da Retinopatia Diabética que tenham realizado o rastreio da DMI, com resultados de rastreio positivo de ambliopia e de DMI, respetivamente, são "referenciados para uma consulta de oftalmologia no SNS, num centro de referência com capacidade diagnóstica e terapêutica, num prazo máximo de quatro semanas, de acordo com o ACES em que se encontram inscritos" (SNS, 2016) ${ }^{\text {a }}$. O Governo planeia ter, em 2020, 28 Agrupamentos de Centros de Saúde (ACES) com rastreio oftalmológico (Público, 2016)a .

Considera-se, todavia, também importante o Rastreio da ambliopia na adolescência, em parceria com as Escolas, aos 15 anos, altura em que é frequente iniciar-se o desenvolvimento de algumas patologias com elevada prevalência, como é o caso da miopia.

Sugere-se ainda o alargamento do Rastreio da DMI à Medicina do Trabalho, em indivíduos ativos, com mais de 55 anos. Poderá ser também promovido o mesmo Rastreio em Unidades de Cuidados Continuados e pelas autarquias, usando as farmácias comunitárias como parceiros de divulgação e motivação para a iniciativa de indivíduos reformados.

Para além destas medidas preventivas, há que realçar o reforço do Programa de Rastreio da Retinopatia Diabética, pela Administração Regional de Saúde de Lisboa e Vale do Tejo (ARSLVT), a cerca de 60.000 Diabéticos desta Região, onde todos os diabéticos serão convocados anualmente pelo seu Centro de Saúde para realização de retinografia. 
Os quatro Agrupamentos de Centros de Saúde (ACES) abrangidos nesta $1^{\text {a }}$ fase são:

- ACES Estuário do Tejo (16.000 Diabéticos)

- ACES Lisboa Ocidental e Oeiras (15.000 Diabéticos)

- ACES Lisboa Norte (15.000 Diabéticos)

- ACES Lisboa Central (17.000 Diabéticos)

A ARSLVT prevê que, no final de 2016, estes rastreios sejam alargados a outros quatro ACES (ACES Sintra; ACES Loures-Odivelas; ACES Amadora e ACES Cascais), aumentando a cobertura a cerca de mais 58.000 diabéticos. No total, no final de 2016, a Região LVT terá rastreado cerca de 118.000 utentes diabéticos (50\% dos Diabéticos inscritos nos Centros de Saúde da Região) (DGS, 2016)e .

Outro exemplo a salientar é o do Hospital Garcia de Orta, que acaba de abrir uma nova unidade, o Núcleo de Oftalmologia de Almada (NOA), preparado para a realização de consultas de oftalmologia e rastreio da retinopatia diabética, glaucoma e despiste da ambliopia na criança na Unidade funcional do ACES Almada Seixal, a cerca de 350 mil habitantes (DGS, 2016)e. O NOA está preparado para disponibilizar os seguintes Meios Complementares de Diagnóstico e Terapêutica (MCDT): retinografia do fundo ocular, paquímetria de contacto, tomografia de coerência ótica maculare do nervo ótico e avaliação ortóptica no contexto da consulta de oftalmologia pediátrica (DGS, 2016)e.

\section{MONITORIZAÇÃO DO IMPACTO DAS POLÍTICAS:}

Para monitorizar o impacto destas políticas é necessário avaliar a redução de CPOD (dentes cariados, perdidos e obturados na dentição definitiva) nas crianças, por exemplo, e o número de casos de DMI e de Retinopatia Diabética evitados e tratados.

Como tal, fez-se uma análise dos indicadores de saúde oral e visual monitorizados pelo Instituto Nacional de Estatística (INE) e pela Direção Geral de Saúde, associados ao Plano Nacional de Saúde (PNS) em vigor. Os resultados encontrados descrevem-se na Tabela 1.

Tabela 1: Indicadores de Saúde Oral e Visual presentemente monitorizados no INE e DGS

\begin{tabular}{|c|c|c|}
\hline Fonte & $\begin{array}{c}\text { Indicadores de } \\
\text { Saúde Oral }\end{array}$ & $\begin{array}{l}\text { Indicadores de } \\
\text { Saúde Visual }\end{array}$ \\
\hline \multirow[t]{3}{*}{$\begin{array}{c}\text { INS } 2014 \text { (INE, } \\
2016)\end{array}$} & $\begin{array}{l}\text { População residente com } 15 \text { ou mais anos por sexo, grupo } \\
\text { etário e momento da última consulta com dentista, NUTS II, } \\
2014\end{array}$ & \multirow[t]{3}{*}{ Não encontrado } \\
\hline & $\begin{array}{l}\text { \% - Proporção da população residente com } 15 \text { ou mais anos } \\
\text { por sexo, grupo etário e momento da última consulta com } \\
\text { dentista, NUTS II, } 2014\end{array}$ & \\
\hline & $\begin{array}{l}\text { População residente com } 15 \text { ou mais anos por sexo, nível de } \\
\text { escolaridade e momento da última consulta com dentista, } \\
\text { Portugal, } 2014\end{array}$ & \\
\hline
\end{tabular}




\begin{tabular}{|c|c|c|}
\hline & $\begin{array}{l}\text { \% - Proporção da população residente com } 15 \text { ou mais anos } \\
\text { por sexo, nível de escolaridade e momento da última } \\
\text { consulta com dentista, Portugal, } 2014\end{array}$ & \\
\hline & $\begin{array}{l}\text { População residente com } 15 \text { ou mais anos que consultou um } \\
\text { dentista nos } 12 \text { meses anteriores à entrevista por sexo, } \\
\text { grupo etário e razão principal da última consulta com } \\
\text { dentista, Portugal, } 2014\end{array}$ & \\
\hline & $\begin{array}{l}\text { \% - Proporção da população residente com } 15 \text { ou mais anos } \\
\text { que consultou um dentista nos } 12 \text { meses anteriores à } \\
\text { entrevista por sexo, grupo etário e razão principal da última } \\
\text { consulta com dentista, Portugal, } 2014\end{array}$ & \\
\hline & $\begin{array}{l}\text { População residente com } 15 \text { ou mais anos que consultou um } \\
\text { dentista nos } 12 \text { meses anteriores à entrevista por sexo, nível } \\
\text { de escolaridade e razão principal da última consulta com } \\
\text { dentista, Portugal, } 2014\end{array}$ & \\
\hline \multirow{4}{*}{$\begin{array}{c}\text { III Estudo } \\
\text { Epidemiológico } \\
\text { Nacional das } \\
\text { Doenças Orais } \\
2012-2014 \\
(\mathrm{DGS}, 2016)^{\mathrm{b}}\end{array}$} & $\begin{array}{l}\text { Índice CPOD (dentes cariados, perdidos e obturados) aos } 12 \\
\text { anos }\end{array}$ & \multirow[t]{4}{*}{-} \\
\hline & $\begin{array}{l}\text { Percentagem de jovens com gengivas saudáveis aos } 12 \text { e } 18 \\
\text { anos }\end{array}$ & \\
\hline & $\begin{array}{l}\text { Número médio de dentes com selantes de fissura aos } 12 \text { e } \\
\text { aos } 18 \text { anos }\end{array}$ & \\
\hline & $\begin{array}{l}\text { Percentagem de crianças e jovens que escovam os dentes à } \\
\text { noite, antes de deitar, aos } 6 \text { anos, aos } 12 \text { anos e aos } 18 \text { anos }\end{array}$ & \\
\hline $\begin{array}{l}\text { PNS 2012-2016 } \\
\text { Indicadores e Metas } \\
\text { em Saúde (DGS, } \\
\text { 2013) }\end{array}$ & $\begin{array}{l}\text { Índice de CPOD (dentes cariados, perdidos e obturados na } \\
\text { dentição definitiva) aos } 12 \text { anos }\end{array}$ & Não encontrado \\
\hline \multirow{2}{*}{$\begin{array}{l}\text { A saúde dos } \\
\text { Portugueses. } \\
\text { Perspetiva 2015 } \\
\text { (DGS, 2015) }\end{array}$} & $\begin{array}{l}\text { Prevalência e gravidade da cárie dentária na dentição } \\
\text { decídua ( } 6 \text { anos) e na dentição permanente ( } 12 \text { anos) }\end{array}$ & \multirow[t]{2}{*}{ Não encontrado } \\
\hline & $\begin{array}{l}\text { Percentagem de jovens com pelo menos } 1 \text { selante de fissura } \\
\text { aplicado em dentes permanentes }\end{array}$ & \\
\hline
\end{tabular}
Fonte: Elaboração Própria

Estes resultados indicam que os indicadores para monitorização da saúde oral no PNS 20122016-2020 são diferentes dos indicadores de base populacional monitorizados no último Inquérito Nacional de Saúde (INS), realizado em 2014, e que os indicadores não são complementares.

No INS 2014 (INE, 2016), os indicadores relacionam-se com o perfil do acesso à medicina dentária para o grupo populacional de maiores de 15 anos.

Os Indicadores associados ao PNS (DGS, 2013 e 2015), focam-se principalmente na prevalência de cáries, maioritariamente aos 12 anos de idade, sem que seja monitorizada qualquer outra patologia do âmbito da estomatologia.

Um cenário semelhante ao anterior passa-se no Estudos Epidemiológico Nacional da Saúde Mental, que, embora mais completo, destaca a prevalência de cáries, de selantes de fissura, de gengivas saudáveis e a adoção de hábitos de cariz preventiva, como a escovagem dos dentes. Quanto a Indicadores de Saúde Visual, o caso é bastante mais grave, uma vez que como não há histórico de monitorização de indicadores de saúde visual em nenhum destes documentos, 
Working Paper

Departamento de Ciências Económicas e Empresariais

Universidade Autónoma de Lisboa

não se consegue avaliar o impacto das medidas tomadas em ganhos em saúde visual da população.

\section{CONCLUSÃO}

Em conclusão, entende-se que o Governo tem feito um esforço para a melhoria da cobertura da saúde oral e visual, no SNS, nomeadamente apostando na prevenção da doença ao nível da boca e da visão. É importante continuar este trabalho e o investimento na cobertura faseada do território até que a saúde oral e a saúde visual alcancem a universalidade e a equidade que se pretendem para o SNS e que está ainda longe de ser alcançada. É ainda preciso constatar que não se encontraram as projeções do custo destas intervenções fase por fase, que seriam interessantes para avaliação e gestão orçamental.

Pretende-se portanto, num médio prazo, o alargamento das medidas de prevenção em saúde oral e visual, que agora se iniciaram, a toda a população, com rastreio da ambliopia e higienização oral para todos, servindo estes cuidados como porta de entrada para acesso ao SNS.

Entende-se que as atividades de promoção da saúde oral e visual nas escolas e na medicina do trabalho são também de extrema importância, devendo ser organizadas a nível nacional pela DGS (por exemplo, organização de campanhas "Aprender a manter os dentes limpos" e "Como proteger a visão") e implementadas pelas ARS (a nível regional) e ACES/ULS (a nível local).

Por último, acredita-se que apostar no tratamento e na celeridade do trabalho em rede relativamente ao tempo de acesso a cuidados especializados após diagnóstico de problemas nos rastreios visuais e na higienização oral, realizados nos CSP, é também essencial.

Crê-se que é interessante haver disponibilidade de consultórios, nas USF, devidamente equipados para oftalmologistas e estomatologistas/ médicos dentistas poderem trabalhar a tempo integral, nos CSP. Sugere-se assim uma alteração do modelo de funcionamento dos CSP e uma deslocalização da maior parte destes especialistas para os ACES/ ULS, para maior proximidade com a população.

\section{REFERÊNCIAS BIBLIOGRÁFICAS}

ARSLVT (2016). ARSLVT reforça Programa de Rastreio da Retinopatia Diabética a cerca de 60.000 Diabéticos da Região. Recuperado em 15 de Outubro de 2016, de http://www.arslvt.min-saude.pt/frontoffice/pages/342?news_id $=750$ 
DGS (2013) PNS 2014-2016 Indicadores e Metas em Saúde. Recuperado a 16 de Outubro de 2016, de http://pns.dgs.pt/files/2012/02/99_7_Indicadores_e_Metas_em_Saude_2013-0118.pdf

DGS (2015). A saúde dos Portugueses. Perspetiva 2015. Recuperado a 16 de Outubro de 2016 , de https://www.dgs.pt/estatisticas-de-saude/estatisticas-de-saude/publicacoes/a-saude-doscaportugueses-perspetiva-2015.aspx

DGS (2016)a ${ }^{\text {a }}$ Programas de saúde prioritários. Recuperado em 5 de Outubro de 2016, de https://www.dgs.pt/programas-de-saude-prioritarios.aspx;

DGS (2016) ${ }^{\mathrm{b}}$. III Estudo Nacional de Prevalência das Doenças Orais. Recuperado em 5 de Outubro de 2016, de https://www.dgs.pt/em-destaque/iii-estudo-nacional-de-prevalencia-dasdoencas-orais-.aspx

DGS (2016) c. Empresários do café apoiam saúde. Recuperado em 5 de Outubro de 2016, de https://www.dgs.pt/em-destaque/empresarios-do-cafe-apoiam-saude.aspx

DGS (2016) d. DGS propõe redução da quantidade de açúcar nas embalagens individuais. Recuperado a 5 de Outubro de 2016, de https://www.dgs.pt/em-destaque/dgs-propoereducao-da-quantidade-de-acucar-nas-embalagens-individuais-.aspx

DGS (2016)e. Núcleo de Oftalmologia de Almada abre aos utentes. Recuperado a 5 de Outubro de 2016, de https://www.dgs.pt/em-destaque/nucleo-de-oftalmologia-de-almada-abre-aosutentes.aspx

DGS (2016) $)^{\ddagger}$. Redução do consumo de açúcar em Portugal. Recuperado em 5 de Outubro de 2016, de https://www.dgs.pt/em-destaque/reducao-do-consumo-de-acucar-em-portugal.aspx EAPN Portugal (2016). Indicadores de pobreza: Dados Europeus e Nacionais Eurostat 2015. Recuperado em 15 de Outubro de 2016, de http://www.eapn.pt/documento/531/indicadoressobre-pobreza-dados-europeus-e-nacionais

Gabinete do Secretário de Estado Adjunto e da Saúde (2016). Despacho 5868-B/2016, de 2 de Maio - Programa Nacional para a Saúde da Visão (PNSV): Rastreio de Saúde Visual Infantil (RSVI) e Rastreio Oportunístico da Degenerescência Macular da Idade (DMI). Diário da República n.ำ 84/2016, 1ํ Suplemento, Série II de 2016-05-02.

Genro, V. (2016). Rastreio da retinopatia diabética. Recuperado em 15 de Outubro de 2016, de http://www.mydiabetes.pt/opini\%C3\%A3o/267-rastreio-da-retinopatia-diab\%C3\%A9tica.html INE (2016). INS 2014. Recuperado em 16 de Outubro de 2017, de https://www.ine.pt/ xportal/xmain?xpid=INE\&xpgid=ine_publicacoes\&PUBLICACOESpub_boui=263714091\&PU BLICACOESmodo $=2$

Jornal Médico $(2016)^{a}$. Consultas de Saúde Oral e de Oftalmologia nos centros de saúde. Recuperado em 15 de Outubro de 2016, de http://www.jornalmedico.pt/2016/02/25/consultasde-saude-oral-e-de-oftalmologia-nos-centros-de-saude/

Jornal Médico $(2016)^{\mathrm{b}}$. APDP disponível para apoiar o Estado em rastreios de retinopatia diabética a nível nacional. Recuperado em 15 de Outubro de 2016, de 
http://www.jornalmedico.pt /2016/10/06/apdp-disponivel-para-apoiar-o-estado-em-rastreiosde-retinopatia-diabetica-a-nivel-nacional

Público (2016)a . Governo quer passar a ter exames e análises nos centros de saúde. Recuperado em 15 de Outubro de 2016, de https://www.publico.pt/sociedade/noticia/governo-quer-passara-ter-exames-e-analises-nos-centros-de-saude-1745023

Público $(2016)^{b}$. Avaliar problemas de visão custa 28 euros por criança. Pais não pagam porque é no SNS. Recuperado em 15 de Outubro de 2016, de https://www.publico.pt/sociedade /noticia/avaliar-problemas-de-visao-custa-28-euros-por-crianca

República Portuguesa (2016)a. Relatório do Orçamento de Estado 2017. Recuperado em 19 de Outubro de 2016, de http://app.parlamento.pt/webutils/docs/doc.pdf?path=61485 23063446f764c3246795a5868774dd546f334e7a67774c336470626d6c7561574e7059585270 646d467a4c31684a53556b76644756346447397a4c33427762444d334c56684a53556c664d 6a49756347526d\&fich=ppl37-XIII_22.pdf\&Inline=true

SNS (2016) ${ }^{\text {. }}$ Rastreio visual infantil arranca em centros de saúde e hospitais da região Norte. Recuperado em 15 de Outubro de 2016, de https://www.sns.gov.pt/noticias/2016 /05/03/rastreio-visual-infantil

SNS (2016) ${ }^{\text {b }}$ Saúde oral nos centros de saúde. Recuperado a 15 de Outubro de 2016, de https://www.sns.gov.pt/noticias/2016/07/04/saude-oral-nos-centros-de-saude/ 